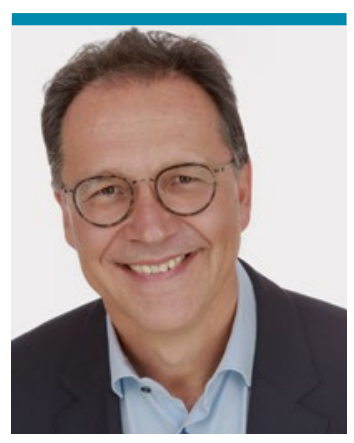

Prof. Dr. Ludger Klimek, Zentrum für Rhinologie und Allergologie Wiesbaden, Präsident des AeDA

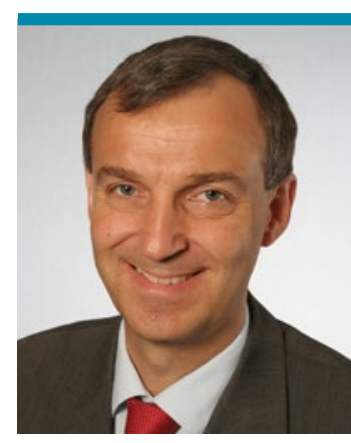

Prof. Dr. Thomas Werfel, Medizinische Hochschule Hannover Präsident der DGAKI

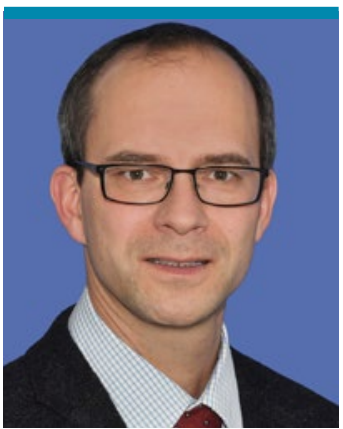

Prof. Dr. Christian Vogelberg, Universitätsklinikum Carl Gustav Carus, Dresden, 1. Vorsitzender der GPA

\title{
Gemeinsam die Allergologie stärken! Forschung - Fortbildung - Versorgung
}

W ir dürfen Sie hiermit herzlich einladen zum 12. Deutschen Allergiekongress (DAK), der vom 5. bis 7. Oktober 2017 in Wiesbaden stattfinden wird. In den historischen Ballsälen des Kurhauses lässt es sich tagen wie in kaum einem anderen Kongresszentrum in Deutschland. „Das schönste Kurhaus der Welt!", schwärmte Kaiser Wilhelm II bereits bei der Eröffnung im Jahre 1907. Gemeinsam mit dem Kurpark, dem „Bowling Green“ und dem hessischen Staatstheater ist es eingebettet in die historischen Gebäude der Altstadt von Wiesbaden in Jugendstil, Barock und Klassizismus: Ein Kongress im Herzen der Stadt!

Mit der gemeinsamen Ausrichtung des DAK zeigen der Ärzteverband Deutscher Allergologen (AeDA), die Gesellschaft für Pädiatrische Allergologie und Umweltmedizin (GPA) und die Deutsche Gesellschaft für Allergologie und klinische Immunologie (DGAKI) ihr gemeinsames Einstehen für eine starke Allergologie in Deutschland.
Das Motto des DAK zeigt dabei, worauf es uns bei dieser Tagung ankommt: Hohe Wissenschaft und neueste Erkenntnisse aus den allergologischen Laboren dieser Welt nutzbar zu machen für Ihre praktische Fortbildung und die Versorgung unserer Patienten. Sie dürfen sich auf eine Fortbildungsveranstaltung auf höchstem Niveau und am Puls der wissenschaftlichen Entwicklung freuen. Alle relevanten Neuentwicklungen der klinischen Allergologie finden ihren Platz im Kongressprogramm.

Wirklich handfest und praktisch wird es dann in einer ganzen Reihe interdisziplinärer Workshops zu sämtlichen allergologischen Themen. In nur drei Tagen können Sie sich über wichtige und aktuelle Neuerungen für den praktischen Alltag in allen Bereichen der Allergologie informieren. Die Vielfalt der Themen ist umfassend: Die interdisziplinäre Allergologie wird komplett dargestellt-vonNahrungsmittelallergien über Asthma bronchiale, Anaphylaxie, Rhinitis, Sinusitis/Polyposis nasi, Urti- karia, Atopisches Ekzem, Angioödeme und Arzneimittelallergien bis hin zur Insektengiftallergie.

Sowohl Neueinsteiger als auch erfahrene Allergologen werden vom interaktiven und interdisziplinären Austausch beim 12. DAK profitieren. Sie finden hier ideale Bedingungen zum wissenschaftlichen und fachlichen Networking: Treffen Sie alte und neue Freunde, die mit Ihnen die Begeisterung für die Allergologie teilen. Wo sonst haben Sie die Gelegenheit, über die Grenzen des eigenen Faches hinauszuschauen und praktische Erfahrungen in „Handson"-Veranstaltungen auch außerhalb Ihrer eigenen Fachdisziplin zu sammeln?

Nutzen Sie das vielfältige Programm des Kongresses und lassen Sie sich von der Allergologie neu begeistern!

Freuen Sie sich schon heute auf ein wissenschaftlich hochkarätiges Programm, kompakte Weiterbildung und ein Wiedersehen mit Freunden und Kollegen beim 12. DAK in Wiesbaden!
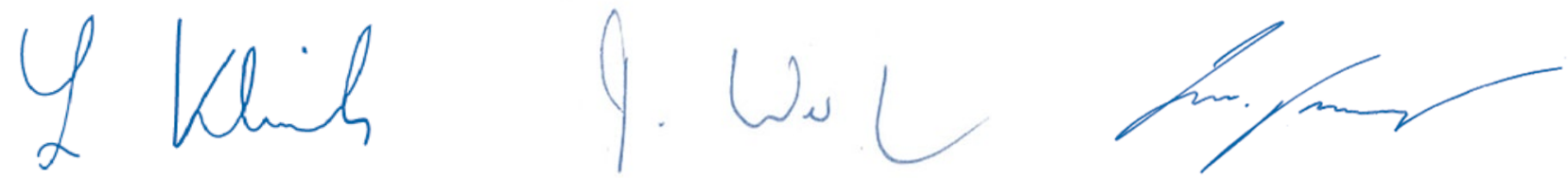